\title{
The new PV prescription for IR singularities of NLO splitting functions
}

\author{
M. Skrzypek ${ }^{\star a}$, O. Gituliar ${ }^{b}$, S. Jadach ${ }^{a}$, A. Kusina ${ }^{c}$ \\ ${ }^{a}$ Institute of Nuclear Physics, Polish Academy of Sciences, \\ ul. Radzikowskiego 152, 31-342 Cracow, Poland \\ ${ }^{b}$ DESY, Platanenallee 6, D-15738 Zeuthen, Germany \\ ${ }^{c}$ Southern Methodist University, Dallas, TX 75275, USA \\ E-mail: Maciej.Skrzypek@ifj.edu.pl, Oleksandr.Gituliar@desy.de, \\ Stanislaw.Jadach@ifj.edu.pl, akusina@smu.edu
}

In this note we outline the Monte Carlo project KrkMC. The goal of this project is to construct a QCD Parton Shower accurate to NLO level in both coefficient function and splitting function (shower) parts. We discuss in detail one of its aspects - the evolution kernels. The kernels had to be recalculated in a new regularisation scheme, called NPV. In this scheme all the singularities in the plus component of the integration momenta are regularised by means of principal value prescription. This is in contrast to the standard approach, in which only the spurious axial singularities are regularised by principal value. As a result, the triple poles in the dimensional regularisation parameter $\varepsilon$ are replaced by a combination of $\varepsilon$-poles and logarithms of geometrical cut-off $\delta$. The resulting exclusive parton densities are more suitable for stochastic applications in four dimensions. Simultaneously, at the inclusive level, the standard and new prescriptions give the same results provided appropriate real and virtual contributions are added.

Loops and Legs in Quantum Field Theory - LL 2014,

27 April - 2 May 2014

Weimar, Germany

${ }^{*}$ Speaker. 


\section{Introduction}

Recently the LHC collaborations managed to demonstrate that Higgs boson decays to fermions are as predicted by the Standard Model [1]. These results mark the onset of the era of "precision Higgs physics" [2], already before the beginning of LHC Run 2, scheduled for early 2015. Such spectacular experimental results call for adequate theoretical tools for stochastic simulations of $p p$ collisions, ultimately to NNLO accuracy. The development of Monte Carlo parton showers started in the mid 1980-ies with the (improved) LO shower and hard processes [3, 4]. It took almost twenty years, until the mid 2000-s, for the next step: hard processes upgraded to NLO precision, while retaining the LO shower [5-7], and this is the present day state-of-the-art solution. For comparison; the analytical inclusive results reached the NNLO precision ten years ago [8-11].

\section{The KrkMC project}

There are a number of promising ideas on how to improve the precision of parton showers [12-17]. We will focus here on the KrkMC project, developed in Kraków [18-23]. Its goal is to propose a new, complete scheme of NLO shower and NLO hard processes. Let us outline briefly its major features. It is based on collinear factorization [24-27], which provides a solid field-theoretical basis. Of course, the collinear factorization is "inclusive"; the transverse degrees of freedom are integrated out. Therefore the new scheme requires:

- Reformulation of collinear factorization theorem in a fully exclusive way.

- Recalculation of the evolution kernels: in an exclusive way, in four dimensions, with well defined relation to $\overline{\mathrm{MS}}$ kernels.

- Construction of kinematical mappings to the true phase space without any gaps or overlaps.

- Formulation of reweighting procedure, reasonably convergent, with positive weights.

As an illustration, let us present a general formula used as a basis of the new NLO scheme; both for the case of upgrading hard matrix elements as well as showers. We show the formulae in a schematic way and we refer to $[18,28]$ for details. In both cases, the starting point is the LO Monte Carlo, that we represent symbolically as (only one hemisphere, $B$ - backward, is shown)

$$
\sigma(s)=\int d x d \sigma_{0}(x s, \hat{\theta}) e^{-S_{I S R}}\left[\delta_{x=1}+\sum_{n=1}^{\infty}\left(\prod_{i=1}^{n} \int_{Q>a_{i}>a_{i-1}} d^{3} \eta_{i} \rho_{1 B}^{(1)}\left(k_{i}\right)\right) \delta_{x=\prod_{j=1}^{n} x_{j}}\right]
$$

where the $\rho$ function is the LO distribution. The inclusion of the NLO correction to the hard process is done by means of a simple weight

$$
W_{M C}^{N L O}=1+\Delta_{S+V}+\sum_{j \in B} \frac{\tilde{\beta}_{1}\left(\hat{s}, \hat{p}_{F}, \hat{p}_{B} ; a_{j}, z_{B j}\right)}{\bar{P}\left(z_{B j}\right) d \sigma_{0}(\hat{s}, \hat{\theta}) / d \Omega}+\{B \leftrightarrow F\}
$$

where $\bar{P}(z) \equiv \frac{1}{2}\left(1+z^{2}\right)$ and

$$
\tilde{\beta}_{1}\left(\hat{p}_{F}, \hat{p}_{B} ; q_{1}, q_{2}, k\right)=\frac{(1-\beta)^{2}}{2} \frac{d \sigma_{0}}{d \Omega_{q}}\left(\hat{s}, \theta_{B 2}\right)-\theta_{\alpha<\beta} \frac{1+(1-\alpha-\beta)^{2}}{2} \frac{d \sigma_{0}}{d \Omega_{q}}(\hat{s}, \hat{\theta})+\{B \leftrightarrow F\}
$$


is the IR- and collinear-finite real emission part and $\Delta_{V+S}$ is a constant virtual+soft correction. The inclusion of the NLO $C_{F}^{2}$-type corrections in the shower is also done by means of a simple weight

$$
\bar{W}_{M C}^{C_{F}^{2}}=1+\sum_{p=1}^{n} \beta_{0}^{(1)}\left(z_{p}\right)+\sum_{p=2}^{n} \sum_{j=1}^{p-1} W\left(\tilde{k}_{p}, \tilde{k}_{j}\right)
$$

The double sum represents summation over the whole cascade of the real-real corrections $W$ that depend on two four-momenta, see [28] for details. The single sum of $\beta_{0}$-functions corresponds to real-virtual contributions. The rest of this note will be devoted to calculation of these real-virtual contributions to the $P_{q q}$ kernel, see also [29-32].

\section{PV prescription}

In the collinear factorization the use of the axial gauge is instrumental. It leads to a transparent physical picture of the evolution and allows for its interpretation in the language of the parton shower. On the other hand though, the axial gauge introduces spurious (unphysical) singularities at the intermediate steps of the calculations. Namely, the gluon propagator has the form

$$
\frac{1}{l^{2}}\left(g^{\mu v}-\frac{l^{\mu} n^{v}+n^{\mu} l^{v}}{n l}\right)
$$

where $n$ is the light-like gauge vector and the denominator $1 / n l$ is the source of the spurious singularities. Of course, once the full set of diagrams is taken into account these singularities must cancel due to gauge invariance. However at the intermediate steps they need regularisation. The traditional approach [26, 33-35] is to use the principal value prescription:

$$
\left[\frac{1}{n l}\right]_{P V}=\frac{n l}{(n l)^{2}+\delta^{2}(n p)^{2}} .
$$

As discussed in [26] this prescription is more like a "phenomenological rule" than a theorem in QFT. A rigorous prescription has been proposed in [36, 37]. It uses two auxiliary vectors, $n$ and $n^{\star}$. However this scheme is difficult in practical calculations $[38,33,34]$ and, due to "ghosts", it is not suitable for stochastic applications ${ }^{1}$.

\section{New use of PV prescription}

Separate contributions to the NLO non-singlet kernels calculated in the PV prescription have $1 / \varepsilon^{3}$ singularities in dimensional parameter $\varepsilon=2-d / 2$, see $[26,33,34]$. Only once the real-real and real-virtual graphs are combined, these singularities cancel. This situation is not acceptable for the Monte Carlo applications, which must be done in four dimensions. Therefore in [28], we performed a calculation of the real-real contributions in a modified PV scheme in which the $1 / \varepsilon^{3}$ poles

\footnotetext{
${ }^{1}$ The concept of linear denominators, $1 /(n l \pm i 0)$, has been used also in NNLO calculations of rapidity distributions of EW bosons [39] in the context of introducing the Dirac-delta constraint via formula:

$$
\int d^{m} k \delta\left(\frac{k p_{1}}{k p_{2}}-u\right) \rightarrow \int d^{m} k \frac{k p_{2}}{k\left(p_{1}-u p_{2}\right)-i 0}-c . c .
$$
}


were replaced by $(1 / \varepsilon) \ln ^{2} \delta$ terms. In order to fully define this New PV (NPV) prescription we had to discuss the real-virtual corrections as well [40]. Based on these results we have formulated the new prescription as follows:

- Standard PV: regularise only the gluon propagator with PV, regularise all other singularities in (+)-components of integration momenta with dimensional regularisation:

$$
\frac{d^{m} l}{l_{+}^{1-\varepsilon}}, \quad l_{+}=\frac{n l}{n p} .
$$

- NPV: use PV to regularise all singularities of the integrand in (+)-components of integration momenta, both real and virtual, keeping higher order terms in $\varepsilon$ as needed:

$$
\frac{d^{m} l}{l_{+}^{1-\varepsilon}} \rightarrow d^{m} l\left[\frac{1}{l_{+}}\right]_{P V}\left(1+\varepsilon \ln l_{+}+\varepsilon^{2} \frac{1}{2} \ln ^{2} l_{+}+\ldots\right) .
$$

The motivation of this approach is the following. All (+)-singularities cancel in the final expression (kernel) [24, 25]. Therefore it is justified to extend the "phenomenological PV rule" of CurciFurmanski-Petronzio, which is based on analogous cancellation of "spurious" singularities in the final expression.

There is one significant consequence of the new scheme: now all the integrals, including the non-axial (Feynman-type) ones depend on the axial vector $n$ (via the products like $n q \equiv q^{+}$). Consider for example the three-point scalar integral with kinematics $p^{2}=(p-q)^{2}=0$ :

$$
J_{3}^{\mathrm{F}}=\int \frac{d^{m} l}{(2 \pi)^{m}} \frac{1}{l^{2}(q-l)^{2}(p-l)^{2}}
$$

In the standard PV prescription we obtain:

$$
J_{3}^{\mathrm{F}}=C\left(-\frac{1}{\varepsilon^{2}}+\frac{\pi^{2}}{6}\right), \quad C=i \frac{\Gamma(1-\varepsilon)}{(4 \pi)^{2}\left|q^{2}\right|}\left(\frac{4 \pi}{\left|q^{2}\right|}\right)^{-\varepsilon}
$$

whereas in the NPV prescription the result is more complicated:

$$
J_{3}^{\mathrm{F}}=C\left(-\frac{1}{\varepsilon}(-2 \ln \delta+\ln (1-x))+2 \ln ^{2} \delta-2 \ln \delta \ln (1-x)+\frac{1}{2} \ln ^{2}(1-x)\right) .
$$

Note, that the singularity $1 / \varepsilon^{2}$ has been replaced by $(1 / \varepsilon) \ln \delta$ and $\ln ^{2} \delta$.

\section{Exclusive contributions to $P_{q q}$ kernel}

In this section we will present the exclusive real-virtual NLO contributions to the evolution kernel $P_{q q}$, obtained in the NPV scheme. Let us start with a brief reminder on how the kernels are calculated. The main idea of collinear factorization is that the contributing graphs can be grouped in a ladder-like structure of objects, $K$; which are "two-particle-irreducible" (axial gauge is instrumental here). In the LO approximation the $K$ are just single emission graphs and the whole structure is a genuine ladder. At the NLO level, among others the real-virtual-type graphs contribute to $K$, see Fig. 1 . The (generalized) ladder is then cut into pieces by means of appropriate 


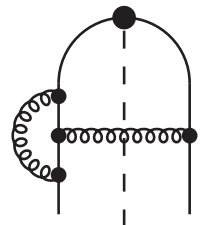

(c) : $C_{F}^{2}-\frac{1}{2} C_{F} C_{A}$

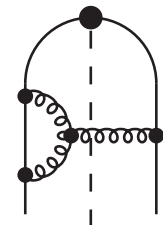

$(d): \frac{1}{2} C_{F} C_{A}$

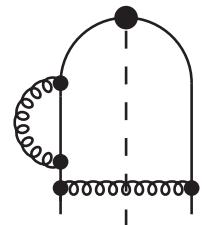

(e) $: \frac{1}{2} C_{F}^{2}$

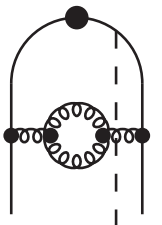

$(f): C_{F} C_{A}$

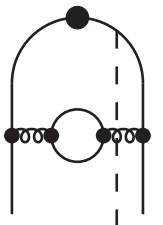

$(g): C_{F} T_{F}$

Figure 1: Real-virtual contributions to NLO non-singlet $P_{q q}$ kernel

projection operators, which extract the singular parts of $K$. In the case of fermions it is done simply by inserting the $\not h$ matrix and taking the trace:

$$
W_{N}=x \operatorname{Tr}\left[\frac{\not h}{4 n q} K \not p\right] .
$$

The $\not h$ matrices are represented by the black dots on top of lines in Fig. 1. The $\not p$ matrices closing fermion lines from below are not shown. In this way we obtain the non-integrated partonic density $W_{N}$. In the next step the integral over virtual loop momenta hidden in $K$ is performed and the renormalisation procedure is carried out. Finally we end up with the exclusive (renormalized) partonic density $W_{R}$, which depends on the four-momentum $k$ of the real emitted parton. Once the real phase space $d \Phi(k)$ is integrated out we obtain the standard inclusive parton densities, $\tilde{\Gamma}$. The evolution kernel $P_{q q}$ is then related to $\tilde{\Gamma}$ as follows (up to terms proportional to $\delta(1-x)$ ):

$$
\tilde{\Gamma}_{q q}(x, \varepsilon)=\delta(1-x)+\frac{1}{\varepsilon}\left(\frac{\alpha_{S}}{2 \pi} P_{q q}^{L O}(x)+\frac{1}{2}\left(\frac{\alpha_{S}}{2 \pi}\right)^{2} P_{q q}^{N L O}(x)+\ldots\right)+\mathscr{O}\left(\frac{1}{\varepsilon^{2}}\right) .
$$

Let us now present the complete exclusive partonic densities $W_{R}$ for the non-singlet $q q$ case in the NPV scheme corresponding to Fig. 1. We group them in two color structures: $C_{F}^{2}$ and $C_{F} C_{A}$ plus $C_{F} T_{F}$ :

$$
\begin{aligned}
& W_{R}^{C_{F}^{2}}=\alpha_{S}^{2} C_{F}^{2} \frac{\Gamma(1-\varepsilon)}{(4 \pi)^{\varepsilon}} \frac{1}{\left|q^{2}\right|}\left\{\frac{1}{\varepsilon} 4 \ln x\left(\left(\frac{\left|q^{2}\right|}{\mu_{R}^{2}}\right)^{\varepsilon}-1\right) P_{q q}\right. \\
& \left.+\left(p_{q q} 4 \operatorname{Li}(1-x)-(1-x)+(1+x)\right)\left(\frac{\left|q^{2}\right|}{\mu_{R}^{2}}\right)^{\varepsilon}\right\}, \\
& W_{R}^{C_{F} C_{A}+C_{F} T_{F}}=\alpha_{S}^{2} C_{F} \frac{\Gamma(1-\varepsilon)}{(4 \pi)^{\varepsilon}} \frac{1}{\left|q^{2}\right|}\left\{\frac{1}{\varepsilon}\left[C_{A} \frac{11}{3}-T_{F} \frac{4}{3}-4 C_{A}\left(\ln (1-x)+I_{0}\right)\left(\frac{\left|q^{2}\right|}{\mu_{R}^{2}}\right)^{\varepsilon}\right] P_{q q}\right. \\
& \left.+4 C_{A}\left[p_{q q}\left(\operatorname{Li}(1)-\operatorname{Li}(1-x)+I_{0} \ln (1-x)-2 I_{1}\right)-\frac{x}{2}\right]\left(\frac{\left|q^{2}\right|}{\mu_{R}^{2}}\right)^{\varepsilon}\right\}, \\
& P_{q q}=p_{q q}+\varepsilon(1-x), \quad p_{q q}=\frac{1+x^{2}}{1-x}, \quad I_{0}=-\ln \delta, \quad I_{1}=-\frac{1}{2} \ln ^{2} \delta-\frac{\pi^{2}}{24}
\end{aligned}
$$

where $q=p-k$ is the momentum of the virtual quark after real emission. As one can see the double poles in $\varepsilon$ vanished, or more precisely, have been replaced by the $\ln \delta$-type terms. This is the main difference with respect to the results in the standard PV scheme known from the literature, and it makes these results "Monte Carlo friendly". 
The exclusive densities $W_{R}^{C_{F}^{2}}$ and $W_{R}^{C_{F} C_{A}+C_{F} T_{F}}$ can be further integrated over the remaining oneparticle phase space. In this way one obtains the inclusive densities, and hence the standard NLO evolution kernel $P_{q q}$. We have verified that in the NPV scheme we reproduce the $\overline{\mathrm{MS}}$ kernel $P_{q q}$ from the literature. Moreover, the inclusive NPV results agree with the PV ones also on a graphby-graph basis, provided the real-real and real-virtual components are added. This forms a strong cross-check of our scheme. Complete results in the NPV scheme, for each graph separately and both exclusive and inclusive, can be found in [30].

\section{The Axiloop package}

In order to facilitate symbolic calculations in the axial (light-cone) gauge we have written a Mathematica package named Axiloop. It is primarily oriented around automated calculation of the NLO kernels. The code is publicly available at http://gituliar.org/axiloop/

Let us list the main features of this package:

- Contains a library of integrals (scalar, vector and tensor), in PV and NPV prescriptions.

- Performs one-loop integration and renormalisation (keeping track of the UV and IR poles).

- Performs one-particle final-state integration.

- Provides auxiliary routines, e.g. for Passarino-Veltman reduction.

As an output one can obtain all mentioned earlier types of density functions (not integrated, bare, exclusive renormalized, inclusive as well as counter-terms).

\section{Summary}

We presented the status of the KrkMC project of constructing QCD Parton Shower in which both the hard matrix element and the shower are upgraded to NLO precision level. We briefly outlined the strategy of the upgrade, and then we discussed in detail one of the components; the evolution kernels that had to be recalculated in a new way.

We presented calculation of all NLO real-virtual one-loop components of the non-singlet $P_{q q}$ kernel in a new, MC-friendly, regularisation scheme, both in inclusive and exclusive forms. The new scheme is based on modified usage of the PV prescription in the light-cone gauge: it applies $\mathrm{PV}$ regularisation to all the singularities in the plus component of the integration momenta.

In the NPV prescription the inclusive NLO kernel $P_{q q}$ agrees with the standard PV one. However, separate real-real and real-virtual contributions differ in PV and NPV: the $1 / \varepsilon^{3}$ poles, present in PV, are replaced by $(1 / \varepsilon) \ln ^{2} \delta$ etc. As a consequence, in the NPV scheme no cancellation of $1 / \varepsilon^{3}$ terms between real and virtual components occurs. Moreover, most of the real graphs are free of higher order poles and due to that can be calculated in four dimensions and can be used for the stochastic simulations.

\section{Acknowledgments}

This work has been partly supported by the Polish National Science Center grants DEC2011/03/B/ST2/02632 and UMO-2012/04/M/ST2/00240, the Research Executive Agency (REA) of the European Union Grant PITN-GA-2010-264564 (LHCPhenoNet), the U.S. Department of Energy under grant DE-FG02-13ER41996 and the Lightner-Sams Foundation. 


\section{References}

[1] CMS Collaboration, S. Chatrchyan et. al., Evidence for the direct decay of the $125 \mathrm{GeV}$ Higgs boson to fermions, Nature Phys. 10 (2014) [arXiv: 1401.6527 ].

[2] CERN Press Release: CERN experiments report new Higgs boson measurements, June 23, 2014.

[3] T. Sjöstrand, A model for initial state parton showers, Phys. Lett. B157 (1985) 321.

[4] B. R. Webber, A QCD model for jet fragmentation including soft gluon interference, Nucl. Phys. B238 (1984) 492.

[5] P. Nason, A new method for combining NLO QCD with shower Monte Carlo algorithms, JHEP 11 (2004) 040, [hep-ph/ 0409146$].$

[6] S. Frixione, P. Nason and C. Oleari, Matching NLO QCD computations with Parton Shower simulations: the POWHEG method, JHEP 0711 (2007) 070, [arXiv: 0709.2092 ].

[7] S. Frixione and B. R. Webber, Matching NLO QCD computations and parton shower simulations, JHEP 06 (2002) 029, [hep-ph/ 0204244$].$

[8] S. Moch, J. A. M. Vermaseren and A. Vogt, The three-loop splitting functions in QCD: The non-singlet case, Nucl. Phys. B688 (2004) 101-134, [hep-ph / 0403192$].$

[9] A. Vogt, S. Moch and J. A. M. Vermaseren, The three-loop splitting functions in QCD: The singlet case, Nucl. Phys. B691 (2004) 129-181, [hep-ph/ 0404111$].$

[10] A. Almasy, S. Moch and A. Vogt, On the Next-to-Next-to-Leading Order Evolution of Flavour-Singlet Fragmentation Functions, Nucl. Phys. B854 (2012) 133-152, [arXiv: 1107 . 2263].

[11] A. Mitov, S. Moch and A. Vogt, Next-to-Next-to-Leading Order Evolution of Non-Singlet Fragmentation Functions, Phys. Lett. B638 (2006) 61-67, [hep-ph / 0604053 ].

[12] S. Tsuno, T. Kaneko, Y. Kurihara, S. Odaka and K. Kato, GR@PPA 2.7 event generator for $p p / p$ anti-p collisions, Comput. Phys. Commun. 175 (2006) 665-677, [hep-ph/ 0602213$].$

[13] S. Höche, F. Krauss, M. Schönherr and F. Siegert, QCD matrix elements + parton showers: The NLO case, JHEP 1304 (2013) 027, [arXiv: 1207 . 5030].

[14] S. Alioli, C. W. Bauer, C. J. Berggren, A. Hornig, F. J. Tackmann, et. al., Combining Higher-Order Resummation with Multiple NLO Calculations and Parton Showers in GENEVA, JHEP 1309 (2013) 120, [arXiv:1211.7049].

[15] K. Hamilton, P. Nason and G. Zanderighi, MINLO: Multi-Scale Improved NLO, JHEP 1210 (2012) 155, [arXiv:1206.3572].

[16] Z. Nagy and D. E. Soper, A parton shower based on factorization of the quantum density matrix, JHEP 1406 (2014) 097, [arXiv: 1401.6364 ].

[17] Z. Nagy and D. E. Soper, Ordering variable for parton showers, arXiv:1401.6366.

[18] S. Jadach, A. Kusina, W. Placzek, M. Skrzypek and M. Slawinska, Inclusion of the QCD next-to-leading order corrections in the quark-gluon Monte Carlo shower, Phys. Rev. D87 (2013) 034029, [arXiv:1103.5015].

[19] M. Skrzypek, S. Jadach, A. Kusina, W. Placzek, M. Slawinska, et. al., Fully NLO Parton Shower in QCD, Acta Phys. Polon. B42 (2011) 2433-2443, [arXiv:1111.5368].

[20] S. Jadach, M. Jezabek, A. Kusina, W. Placzek and M. Skrzypek, NLO corrections to hard process in QCD shower - proof of concept, Acta Phys. Polon. B43 (2012) 2067, [arXiv: 1209.4291$].$ 
[21] S. Jadach, A. Kusina, W. Placzek and M. Skrzypek, NLO corrections in the initial-state parton shower Monte Carlo, Acta Phys. Polon. B44 (2013), no. 11 2179-2187, [arXiv: 1310 . 6090].

[22] S. Jadach and M. Skrzypek, QCD evolution in the fully unintegrated form, Acta Phys. Polon. B40 (2009) 2071-2096, [arXiv:0905.1399].

[23] S. Jadach, M. Skrzypek, A. Kusina and M. Slawinska, Exclusive Monte Carlo modelling of NLO DGLAP evolution, PoS RADCOR2009 (2010) 069, [arXiv: 1002.0010 ].

[24] R. K. Ellis, H. Georgi, M. Machacek, H. D. Politzer and G. G. Ross, Perturbation Theory and the Parton Model in QCD, Nucl. Phys. B152 (1979) 285.

[25] R. K. Ellis, H. Georgi, M. Machacek, H. D. Politzer and G. G. Ross, Factorization and the Parton Model in QCD, Phys. Lett. B78 (1978) 281.

[26] G. Curci, W. Furmanski and R. Petronzio, Evolution of parton densities beyond leading order: the non-singlet case, Nucl. Phys. B175 (1980) 27.

[27] J. C. Collins, D. E. Soper and G. Sterman, Transverse momentum distribution in Drell-Yan pair and W and Z boson production, Nucl. Phys. B250 (1985) 199.

[28] S. Jadach, A. Kusina, M. Skrzypek and M. Slawinska, Two real parton contributions to non-singlet kernels for exclusive QCD DGLAP evolution, JHEP 08 (2011) 012, [arXiv: 1102 . 5083].

[29] O. Gituliar, S. Jadach, A. Kusina and M. Skrzypek, Calculation of QCD NLO Splitting Functions in the light-cone gauge: a new regularization prescription, arXiv : 1406.4283.

[30] O. Gituliar, Higher-Order Corrections in QCD Evolution Equations and Tools for Their Calculation, arXiv:1403.6897.

[31] O. Gituliar, S. Jadach, A. Kusina and M. Skrzypek, Virtual Corrections to the NLO Splitting Functions for Monte Carlo: the non-singlet case, Acta Phys. Polon. B44 (2013), no. 11 2197-2206, [arXiv:1310.7537].

[32] O. Gituliar and M. Skrzypek, Automatic Calculations of NLO Splitting Functions with Loops for Exclusive Parton Shower Monte Carlo, Acta Phys. Polon. B44 (2013), no. 7 1469-1479.

[33] G. Heinrich, Improved techniques to calculate two-loop anomalous dimensions in QCD. PhD thesis, Swiss Federal Institute of Technology, Zurich, 1998.

[34] G. Heinrich and Z. Kunszt, Two-loop anomalous dimension in light-cone gauge with Mandelstam-Leibbrandt prescription, Nucl. Phys. B519 (1998) 405-432, [hep-ph/ 9708334 ].

[35] R. K. Ellis and W. Vogelsang, The evolution of parton distributions beyond leading order: the singlet case, hep-ph/9602356.

[36] S. Mandelstam, Light Cone Superspace and the Ultraviolet Finiteness of the N=4 Model, Nucl. Phys. B213 (1983) 149-168.

[37] G. Leibbrandt, The Light Cone Gauge in Yang-Mills Theory, Phys. Rev. D29 (1984) 1699.

[38] A. Bassetto, G. Heinrich, Z. Kunszt and W. Vogelsang, The light-cone gauge and the calculation of the two-loop splitting functions, Phys. Rev. D58 (1998) 094020, [hep-ph/9805283].

[39] C. Anastasiou, L. J. Dixon, K. Melnikov and F. Petriello, High-precision QCD at hadron colliders: Electroweak gauge boson rapidity distributions at NNLO, Phys. Rev. D69 (2004) 094008, [hep-ph/0312266].

[40] O. Gituliar, S. Jadach, A. Kusina and M. Skrzypek, On regularizing the infrared singularities in QCD NLO splitting functions with the new Principal Value prescription, Phys. Lett. B732 (2014) 218-222, [arXiv:1401.5087]. 